\title{
Isolation of the Drosophila melanogaster Dunce Chromosomal Region and Recombinational Mapping of Dunce Sequences with Restriction Site Polymorphisms as Genetic Markers
}

\author{
RONALD L. DAVIS*† AND NORMAN DAVIDSON \\ Department of Chemistry, California Institute of Technology, Pasadena, California 91125
}

Received 15 August 1983/Accepted 31 October 1983

\begin{abstract}
Using the method of chromosomal walking, we have isolated a contiguous region of the Drosophila melanogaster $\mathrm{X}$ chromosome which corresponds to salivary gland chromosome bands 3C12 to 3D4. This five-band region contains approximately 100 kilobases of DNA, including those sequences comprising dunce, a gene which functions in memory and cyclic nucleotide metabolism. Genome blots of DNA from flies carrying several different chromosomal aberrations with breakpoints in the region have been probed with the isolated clones to map the breakpoints on the cloned DNA and to delimit dunce sequences. This has localized dunce to a 50-kilobase region. In addition, we have searched this 50-kilobase region for restriction site polymorphisms between $\mathrm{X}$ chromosomes from different Drosophila strains by genome blotting experiments, and we have followed the segregation of detected polymorphisms and dunce alleles after meiotic recombination. The data map one dunce mutation between two polymorphisms located 10 to 12 kilobases apart.
\end{abstract}

The dunce $(d n c)$ gene of Drosophila melanogaster is of special interest because it plays a role in cyclic nucleotide metabolism and a variety of behavioral processes. The $d n c$ mutant flies execute poorly several different associative learning tasks, including those employing olfactory (9) and visual (11) cues, with positive (31) or negative (9) reinforcement. The associative learning deficit is manifest not only in flies but also in dnc mutant larvae (1). Operant conditioning is altered by lesions in this gene (3), as are the nonassociative learning responses of habituation and sensitization (10). In addition, dnc mutations disrupt one aspect of normal courtship behavior (13). Although normal learning by $d n c$ mutants is not detected in some situations (11), the mutants learn normally but forget rapidly in others $(8,10,31)$. Consequently, $d n c$ flies are best classed as memory mutants.

The observation that the $d n c$ mutation perturbs normal cyclic AMP metabolism (4) led to the suggestion that cyclic AMP is intimately involved in behavioral plasticity, a conclusion also reached from studies of learning and memory by Aplysia (15). More specifically, one of three normal forms of cyclic nucleotide phosphodiesterase expressed in normal Drosophila adults $(6,16 ;$ R. L. Davis and L. M. Kauvar, Adv. Cyclic Nucleotide Res., in press) is deficient in $d n c$ mutants $(4,7)$. The current evidence suggests that $d n c$ is the structural gene for this form of cyclic AMP phosphodiesterase (Davis and Kauvar, in press). Cyclic AMP levels are elevated in $d n c$ mutants $(4,7)$, apparently due to the loss in one of the degradative enzymes.

We describe here the isolation of the chromosomal region which contains $d n c^{+}$, and also our experiments to map the gene on cloned DNA as the first step to probe the structure, regulation, evolution, and biological function of the gene. Chromosomal walking has been employed to isolate the $\mathrm{dnc}^{+}$chromosomal region, and principles of recombinational mapping advanced by Sturtevant (30) have been utilized to map dnc sequences on the isolated DNA.

* Corresponding author.

$\dagger$ Present address: Department of Biochemistry, Michigan State University, East Lansing, MI 48824.

\section{MATERIALS AND METHODS}

Nucleic acid isolation. Bacteriophage $\lambda$ DNA was isolated essentially as described by Maniatis et al. (21). Plasmid DNA was isolated by the methods of Ish-Horowicz and Burke (14).

Genome DNA was isolated from adult flies (12). Frozen flies were ground to a fine powder with a pestle in a mortar cooled on dry ice. The powder was suspended in ice-cold $0.35 \mathrm{M}$ sucrose-0.050 M Tris-hydrochloride ( $\mathrm{pH} 7.6)-0.025$ $\mathrm{M} \mathrm{KCl}-0.005 \mathrm{M}$ magnesium acetate, and cell breakage was completed by Dounce homogenization. The homogenate was filtered once or twice through Nitex, and the filtrate was centrifuged at $4^{\circ} \mathrm{C}$ at $4,000 \times g$ for $15 \mathrm{~min}$. The nuclear pellet was suspended in homogenization buffer and recentrifuged. The nuclei were then suspended in $0.15 \mathrm{M} \mathrm{NaCl}-0.10 \mathrm{M}$ EDTA-0.050 M Tris-hydrochloride ( $\mathrm{pH}$ 8.0). Proteinase K was added to a concentration of $20 \mu \mathrm{g} / \mathrm{ml}$, Sarkosyl was added to $2 \%$, and the solution was incubated at $50^{\circ} \mathrm{C}$ for $2 \mathrm{~h}$. Solid $\mathrm{CsCl}$ was added to $p=1.7$, and the DNA was banded by centrifugation for $60 \mathrm{~h}$ at $20^{\circ} \mathrm{C}$ in a Ti50 rotor at 38,000 $\mathrm{rpm}$. The genomic DNA was collected from the side of the tube as a viscous fraction and was dialyzed extensively against $0.010 \mathrm{M}$ Tris-hydrochloride $(\mathrm{pH} 8.0)-0.010 \mathrm{M} \mathrm{NaCl}-$ 0.001 M EDTA.

For rapid analysis of genome restriction sites, 10 adult flies were ground to a fine suspension in a ground-glass microhomogenizer in $0.4 \mathrm{ml}$ of $0.05 \mathrm{M}$ Tris-hydrochloride (pH 8.0)-0.010 M EDTA-0.5\% sodium dodecyl sulfate. Proteinase $\mathrm{K}$ was added to a concentration of $1 \mathrm{mg} / \mathrm{ml}$ and was incubated at $37^{\circ} \mathrm{C}$ for 4 to $20 \mathrm{~h}$. The solution was extracted twice with phenol-chloroform-isoamyl alcohol $(25: 24: 1)$ with incubation of the emulsion at $50^{\circ} \mathrm{C}$ for $15 \mathrm{~min}$ during the extractions. The aqueous phase was extracted with ether, and residual ether was removed under a stream of $\mathrm{N}_{2}$. Sodium chloride was added to $0.3 \mathrm{M}$ with 1 volume of isopropyl alcohol. After precipitation of the nucleic acids in the cold and centrifugation, the pellet was washed with $70 \%$ ethanol, dried under reduced pressure, and suspended in 20 $\mu l$ of $0.010 \mathrm{M}$ Tris-hydrochloride ( $\mathrm{pH} \mathrm{8.0)-0.001} \mathrm{M} \mathrm{EDTA.}$ 
This procedure can be scaled up for larger numbers of flies. For restriction by six-hitters and blotting, we generally used DNA isolated from 10 flies per lane, although we have used DNA from as few as 2 flies. For restriction by four-hitters and blotting, 50 adult flies were used to isolate DNA for one gel lane.

Gel isolation of restriction fragments. Preparative restriction digests were fractionated on horizontal agarose gels and stained with ethidium bromide to visualize the DNA. A trough was cut adjacent to the fragment to be isolated, a strip of DE81 paper was inserted, and the fragment was electrophoresed into the paper; this electrophoresis was perpendicular to the direction used for initial fractionation. After all the DNA was electrophoresed into the paper, the paper was removed, trimmed, and washed extensively with $0.010 \mathrm{M}$ Tris-hydrochloride ( $\mathrm{pH} 8.0)-0.10 \mathrm{M} \mathrm{NaCl}-0.001 \mathrm{M}$ EDTA. The DNA was eluted from the paper with six $100-\mu$ l washes with $0.010 \mathrm{M}$ Tris-hydrochloride (pH 8.0)-1.0 M NaCl-0.001 $M$ EDTA and was filtered through a plug of silanized glass wool. The filtrate was extracted twice with $n$-butanol and once with ether, and residual ether was removed with a stream of $\mathrm{N}_{2}$. An equal volume of isopropyl alcohol was added to precipitate the DNA. After storage at $-20^{\circ} \mathrm{C}$, the DNA was recovered by centrifugation, and the pellet washed with $70 \%$ ethanol, dried, and suspended in $0.010 \mathrm{M}$ Tris-hydrochloride ( $\mathrm{pH} 8.0$ ) $-0.010 \mathrm{M} \mathrm{NaCl}-0.001 \mathrm{M}$ EDTA. Recoveries ranged from 30 to $70 \%$, depending on the fragment size. The DNA recovered was of high quality and could be used in all standard enzymatic reactions.

DNA labeling. Cloned DNA or isolated restriction fragments were labeled by nick translation as described by Mullins et al. (23).

Library screening and blot hybridizations. In general, eight genome equivalents from two different libraries were screened for chromosomal walking. Library screens and blot hybridizations were conducted by procedures described by Mullins et al. (23).

Subcloning. Restriction fragthents from certain $\lambda$ clones were subcloned into plasmid pBR322 or pUC8 by standard procedures.

Restriction digestions. Restrlation enzymes were purchased from New England Biolabs or Bethesda Research Laboratories. The 24 restriction enzymes used which cut infrequently included $A c c \mathrm{I}, B c l \mathrm{I}, B a m \mathrm{HI}, B g l \mathrm{I}, B g l \mathrm{II}$, Bst NI, ClaI, EcoRI, HincII, HindIII, HpaII, KpnI, NaeI, NarI, NruI, PstI, PvuII, SacI, SacII, SalI, SmaI, SphI, StuI, and XhoI. Frequent cutters included AluI, DdeI, HaeII, HaeIII, HhaI, HinfI, HphI, MboI, MboII, MspI, RsaI, Sau96I, TaqI, and ThaI.

Fly strains and crosses. The fly strains utilized here have been described $(7,18)$, except for $D f(1) d m^{77 h}$, which was induced by $\mathrm{G}$. LeFevre in the Amherst wild type. The cytologies of chromosomal aberrations are those of G. LeFevre (personal communication).

To construct dunce region deficiencies, $C(1) D X, y f / w^{+} Y$ ㅇ were crossed with $D f(1) N ; S M I, C y D p(1 ; 2) w^{+51 b 7} \delta$, and the progeny males, $D f(1) N / w^{+} Y$, were selected. The $w^{+} Y$ chromosome covers the hemizygous lethality associated with notch $(N)$ deficiency but does not extend to the $d n c$ locus.

To generate recombinants near the $d n c$ locus, males of the genotype $s c w^{b l} d n c^{2}$ were crossed to $D f(1) d m^{77 h} / F M 7 q$, and $s c w^{b l} d n c^{2} / D f(I) d m^{77 h}$ progeny females further mated to produce recombinant $X$ chromosomes recovered in their male progeny. Stocks were established with attached-X females. The marker scute $(s c)$, although present in one parental chromosome, has been omitted in the text discussion since it played no role in the present analysis.

All $115 w^{+}$-viable recombinant stocks analyzed were examined for fertlale fertility by crossing males from each stock to $D f(1) d m^{75 e 19} / F M 7$ females, selecting five non-FM7 heterozygote progeny, and mating these to males. No intermediate fertility was observed; females from these tests produced very few or no progeny, or else they produced many progeny. These recombinants were then tested for the presence of the insertion element (see Fig. 6). The 20 recombinants which exhibited crossover to the right of this element were assayed for cyclic AMP phosphodiesterase activity by L. Kauvar (16). These recombinants were clearly $d n c^{2}$ or $d n c^{+}$by the enzymatic assay.

\section{RESULTS}

Genetic organization of the chromosomal region containing dunce. Cytogenetic evidence indicates that $d n c$ resides at chromomere 3D4 of the salivary gland X chromosome (Fig. 1). This assignment was made from the observation that the deficiency $D f(1) N^{64 j 15}$ does not completely remove the $d n c^{+}$ function, whereas $D f(1) N^{64 i 16}$ and $D f(1) N^{71 h 24-5}$ do $(7,18$, 26). Since the right breakpoint of $D f(1) N^{64 j 15}$ has been localized between chromomeres 3D3 and 3D4 and the right breakpoints of $D f(1) N^{64 i 16}$ and $D f(1) N^{71 h 24-5}$ have been localized between 3D4 and 3D5, the genetic analyses place $d n c$ in chromomere 3D4. To the left of $d n c$ but still within chromomere 3D4 is a gene named sam, whose normal function is required for sperm motility (26). The next known genetic function to the left of $\operatorname{sam}$ is $S g s-4$, which resides in chromomere 3C11-12 and produces one of the larval glue polypeptides (19).

The breakpoints associated with the chromosomal aberrations $D f(1) d m^{75 e 19}, w^{+} Y$, and $D f(1) d m^{77 h}$ are also of interest for the current analysis and are depicted in Fig. 1. The left breakpoint of $D f(1) \mathrm{dm}^{75 \mathrm{e}^{19}}$ and the right breakpoint of $w^{+} Y$ are both located to the left of $d n c$, as determined by genetic criteria (26). The chromosome $D f(1) d m^{77 h}$ does not remove the $d n c^{+}$function; therefore, sequences removed by this deletion normally flank $d n c$ on the right.

Isolation of the chromosomal region containing dunce ${ }^{+}$. The molecular isolation of the $\$ g s-4$ locus (24) offered an entry point to the chromosomal region containing the $d n c$ gene (Fig. 2). The clones $\lambda c D m 1570$ and $\lambda c D m 1568$ were isolated and characterized as part of $S g s-4$ studies $(22,24)$. In addition, those studies establishted the centromere-telomere orientation of the cloned region. Consequently, we isolated a 4.2-kilobase (kb) SalI fragment from the centrotmere-proximal end of $\lambda \mathrm{cDm} 1568$ as a probe to screen genome libraries, and we initiated a chromosomal walk in a centromeric direction.

Drosophila genome libraries from the strain Canton-S in bacteriophage $\lambda$ vectors $(5,21)$ were screened with the 4.2 $\mathrm{kb}$ Sall fragment and then successively, by using gel-isolated restriction fragments, from the centromere-proximal end of newly isolated clones as probes. At each step, we mapped the newly isolated clones with restriction enzymes. When necessary, we performed blotting experiments to determine the extend of overlap with characterized clones. In addition, qualitative genome blots were performed with each isolated fragment before screening the libraries to ensure that probe sequences were not repetitive in the genome. In this manner, we isolated a contiguous stretch of Drosophila genome DNA residing just proximal to the $S g s-4$ locus on the X chromosome. The restriction map of this region, deduced from 


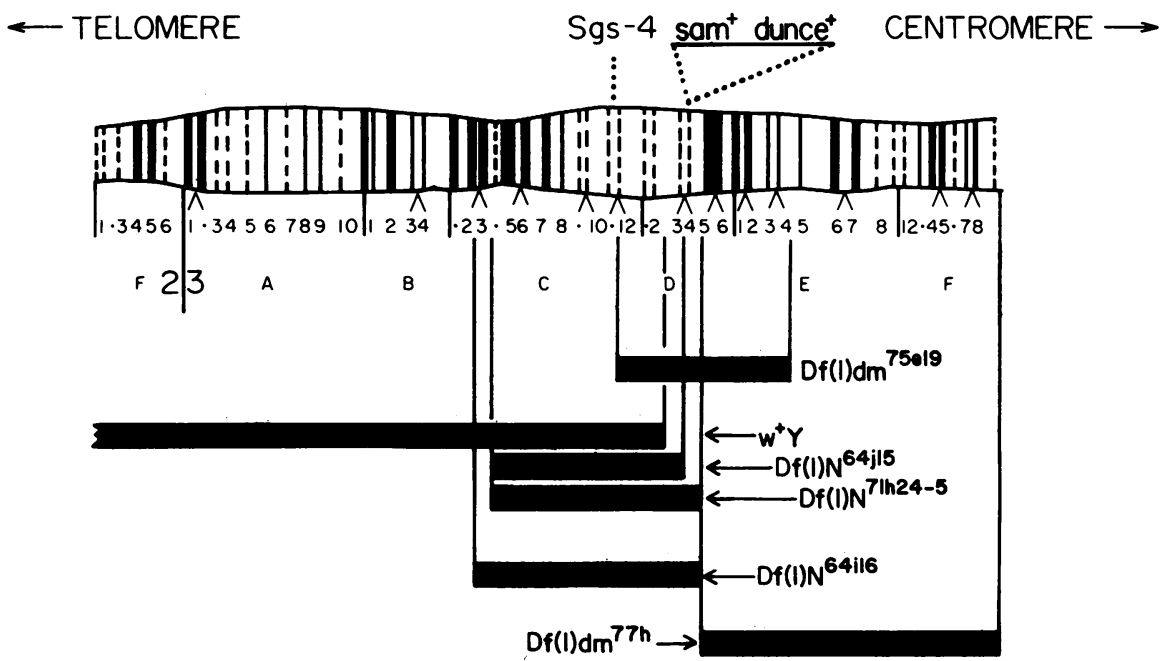

FIG. 1. Cytogenetic organization of the dunce chromosomal region (schematic illustration of a distal region of the $\mathrm{X}$ chromosome). The Sgs-4 locus is in chromomere 3C11 or 12; sam and $d n c$ both reside in 3D4. The cytological extents of several deficiency chromosomes and of $w^{+} Y$, an insertional translocation of $X$ chromosomal region 2D1 through 3D2 into the $Y$ chromosome, are shown below the chromosome. In this latter chromosome, the solid line segment indicates that portion of the $\mathrm{X}$ chromosome which is present in the translocation $\mathrm{Y}$. Cytologies are those of G. LeFevre (personal communication).

analyzing the isolated clones, is depicted in Fig. 2. The extent of some of the clones recovered is also shown.

Mapping breakpoints of chromosomal aberrations. Since breakpoints associated with chromosomal aberrations perturb the restriction map at the site of the breakpoint, their location can be mapped by genome blotting experiments. If chromosomal aberrations have been defined genetically, they can be used to locate critical regions on cloned DNA.
Several chromosomal aberrations breaking near $d n c$ are depicted in Fig. 1. We expected to cross the following breakpoints during the walk in this order: the left breakpoint of $D f(1) \mathrm{dm}^{75 e 19}$, the right breakpoint of $w^{+} Y$, the right breakpoint of $D f(1) N^{64 j 15}$, the right breakpoint of $D f(1) N^{64 i 16}$ and $D f(1) N^{71 h 24-5}$, and the left breakpoint of $D f(1) d m^{77 h}$, the last three being cytologically identical.

Since the $d n c$ region can be completely deleted from the

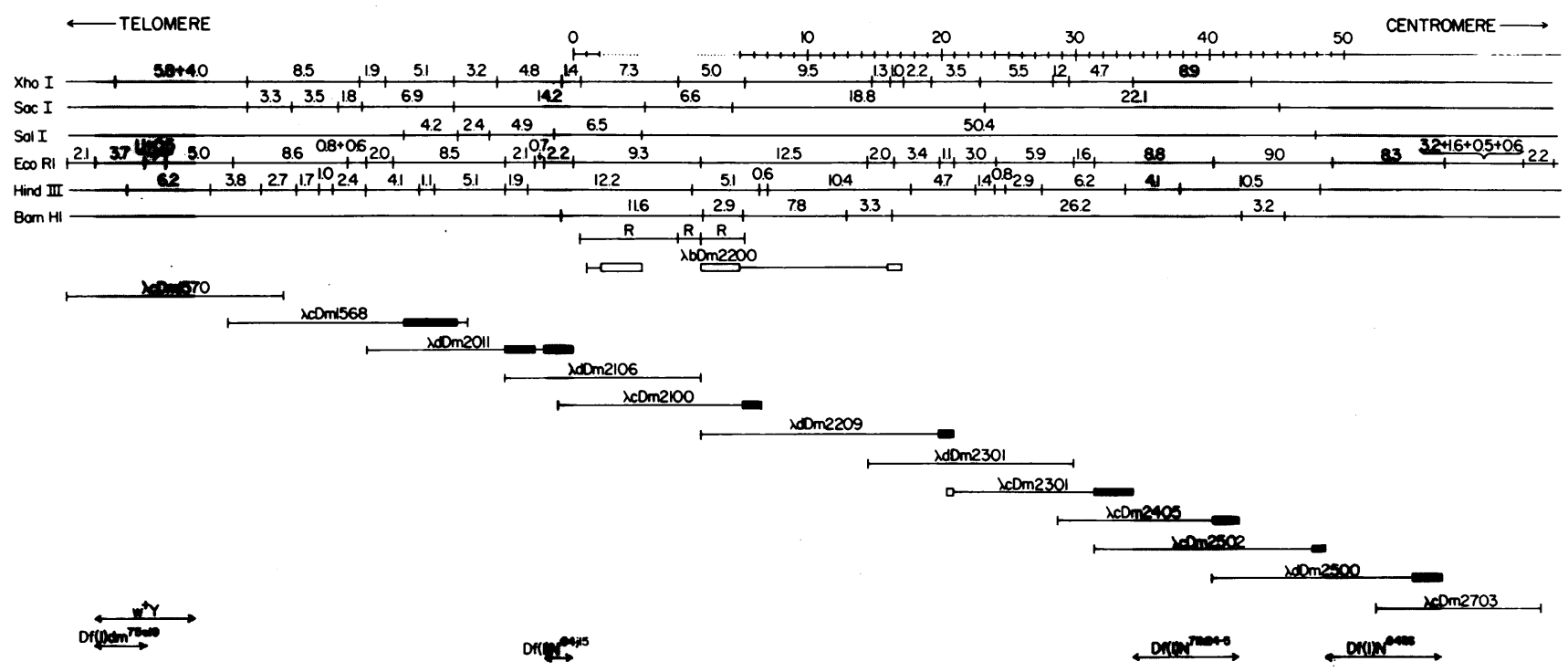

FIG. 2. Restriction map of the dunce chromosomal region (3C12 to 3D4 chromosomal region). Inserts of Drosophila genome DNA in $\lambda$ vectors are shown as line segments below the map. Those isolated from the library prepared by shearing genome DNA and adding EcoRI linkers for cloning (21) are identified with a $\mathrm{c}$ in the nomenclature. Those from the library prepared from genome DNA partially digested with EcoRI (5) have been assigned a d. The blackened segments of certain clones shows the restriction fragments isolated to probe the libraries for each successive step. Open segments indicate uncertainties regarding the endpoints of some clones. The breakpoints of chromosomal aberrations in the region have been mapped (see Fig. 3) and are illustrated here. The widths of the broad shaded areas show our uncertainty from the mapping data. Certain restriction fragments in the region are known to contain repetitive sequences (R). Except for this one repetitive region, the cloned DNA to the right of the $D f(1) N^{64 j 15}$ breakpoint is unique, established by genome blotting experiments. Above the restriction map is the arbitrary coordinate system set up for analyzing restriction site polymorphisms, expressed in kb of unique-sequence DNA from the right site of the 2.2-kb EcoRI fragment of $\lambda \mathrm{dDm} 2106$. Note that the repetitive insertion sequence $(7.3 \mathrm{~kb})$ found in the Canton-S strain between coordinates 2 and 5 is not counted in the coordinate system. 
fly without affecting viability (17), we have used a plusminus (hybridization versus no hybridization) genome blot assay to determine whether cloned probe sequences are present in the DNA of flies genetically constructed to contain various extents of the $d n c$ region. This method circumvents potential problems owing to restriction site polymorphism. McGinnis et al. (22) previously localized the left breakpoint of $D f(1) \mathrm{dm}^{75 e 19}$ and the right breakpoint of $w^{+} Y$. Our data (not shown) map these breakpoints as shown in Fig. 2, confirming the localizations published previously.

The genome blots which establish the positions of the right breakpoints of $D f(1) N^{64 j 15}, D f(1) N^{64 i 16}$, and $D f(1)^{7 / h 24-5}$ are shown in Fig. 3 and are summarized in Fig. 2. The 0.7-kb EcoRI fragment of $\lambda \mathrm{dDm} 2106$ does not hybridize to any of the deficiency chromosomes, whereas a probe containing sequences just to the right (the 2.1- plus 2.2-kb EcoRI fragments) detects a 7.8-kb EcoRI fragment in the genome of flies $D f(1) N^{64 j 15} / w^{+} Y$ (Fig. $3 A$ and $3 B$ ). Other unique-sequence probes to the left of the 2.2-kb EcoRI fragment but to the right of the $w^{+} Y$ breakpoint have not detected homologous sequences in the $D f(I) N^{64 j 15} / w^{+} Y$ genome. This shows that the right breakpoint of $D f(1) N^{64 j 15}$ is within the 2.2-kb EcoRI fragment of $\lambda \mathrm{dDm} 2106$ and that the 7.8-kb homologous fragment in the $D f(1) N^{64 j 15} / w^{+} Y$ genome is a fusion fragment.

Unique-sequence probes to the right of this region through sequences carried by $\lambda \mathrm{cDm} 2301$ do not hybridize to the genomes of $D f(1) N^{54 i l 6 / w^{+}} Y$ and $D f(l) N^{7 / h 24-5} / w^{+} Y$. The probe $\lambda \mathrm{cDm} 2405$ does detect homologous sequences in the $D f(l) N^{7 / h 24-5}$ chromosome (Fig. 3C), indicating that the right breakpoint of this deficiency is between the right ends of $\lambda c D m 2301$ and $\lambda c D m 2405$. The right breakpoint of $D f(l) N^{64 i 16}$ is between the right limits of $\lambda c D m 2502$ and $\lambda \mathrm{dDm} 2500$, as shown by hybridization of $D f(1) N^{64 i l 6} / w^{+} Y$ DNA to $\lambda \mathrm{dDm} 2500$ but not to $\lambda \mathrm{cDm} 2502$. We have searched for the left breakpoint of $D f(l) \mathrm{dm}^{77 h}$ but have not identified it within the interval of DNA which we have isolated.

Since the right breakpoint of $D f(l) N^{64 j l 5}$ is to the left of $d n c$ genetically and the deficiencies $N^{64 i 16}$ and $N^{7 / h 24-5}$ remove $d n c^{+}$activity, these data suggest that the gene resides in the interval of ca. $50 \mathrm{~kb}$ between the breakpoints of $D f(I) N^{64 j I 5}$ and $D f(I) N^{71 h 24-5}$. However, from these data we cannot exclude the possibilities that $D f(1) N^{64 i 16}$, $D f(1) N^{7 l h 24-5}$, or both break within the gene and disrupt its function or that the gene resides to the right of these deficiencies and is structurally intact but inactive due to a position effect (28) of juxtaposed sequences on the gene.

Genomic representation of dunce chromosomal sequences. With one exception, the sequences of interest in the interval of ca. $50 \mathrm{~kb}$ are represented once per haploid genome. This was established by qualitative genome blots. We have probed Canton-S genome blots with a variety of cloned sequences spanning the $50-\mathrm{kb}$ interval and find that the only genome restriction fragments which hybridize to the probes are those predicted by the map of cloned DNA (data not shown). Furthermore, the genome blots with dnc-deficiency DNA (Fig. 3) support this conclusion since certain clones show no hybridization to these genomes. Therefore, it is not possible that the $d n c$ region is duplicated elsewhere in the genome.

The exception is a repetitive sequence encountered during the chromosomal walk which exists entirely within $\lambda c D m 2100$ (Fig. 2). This sequence is repeated between 30 and 50 times in the Canton-S genome, as indicated by probing genome blots with $\lambda \mathrm{cDm} 2100$ or gel isolated restriction fragments (Fig. 2).

We probed an Oregon-R genome library (constructed by E. Meyerowitz) with the unique-sequence $1.3-\mathrm{kb}$ Xho-EcoRI (synthetic) fragment and the 1.4-kb Xho fragment of $\lambda c D m 2100$, fragments which flank the repetitive sequence on the right and left, respectively. One positive identified with the 1.3-kb Xho-EcoRI fragment named $\lambda$ bDm2200 (Fig. 2) was characterized in detail. Restriction mapping and clone blotting experiments reveal that this clone spans the repetitive sequence region; however, genome blotting experiments show that the clone contains only unique sequences (data

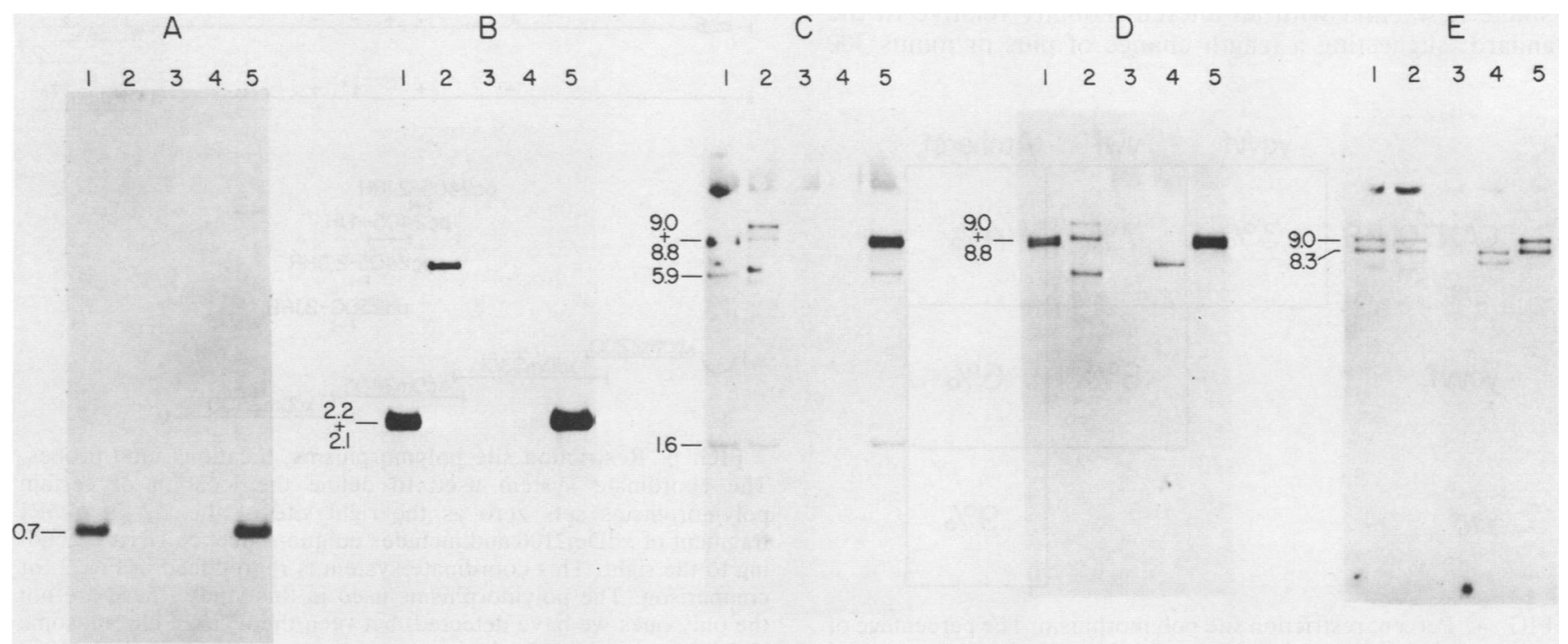

FIG. 3. Genome blots of DNA from dunce deficiencies. EcoRI digests of DNA from male flies of genotypes Canton-S (lanes 1 and 5), $D f(I) N^{\sigma 4 j l 5} / w^{+} Y$ (lanes 2), $D f(I) N^{64 i l 6} / w^{+} Y$ (lanes 3), and $D f(l) N^{7 l h 24-5} / w^{+} Y$ (lanes 4) were fractionated. blotted, and probed with the $0.7-\mathrm{kb}$ $E c o R I$ fragment of $\lambda \mathrm{dDm} 2106(\mathrm{~A})$ and the 2.2 - plus $2.1-\mathrm{kb} E c o$ RI fragments of $\lambda \mathrm{dDm} 2106$ (B), $\lambda \mathrm{cDm} 2405$ (C), $\lambda \mathrm{cDm} 2502$ (D), and $\lambda \mathrm{dDm} 2500$ (E). See the text for the genetic construction of the deficiency flies. The hybridizing restriction fragment in panel C, lane 4 , is very faint in the original autoradiogram and did not reproduce well in photographs. This fragment comigrates with the smaller of the two bands observed in panel D, lane 4 . 
not shown). Probing genome blots of DNAs from different Drosophila strains with $\lambda \mathrm{bDm} 2200$ and other unique-sequence restriction fragments in the vicinity disclose that Canton-S has a 7.3-kb insertion of DNA relative to other strains. It is likely that this is a transposable element since most of the middle repetitive DNA in $D$. melanogaster is nomadic (32).

In summary, these data indicate that chromomere 3D4, which is defined by the breakpoints of $D f(1) N^{64 j 15}$ and $D f(I) N^{7 / h 24-5}$, is composed of approximately $50 \mathrm{~kb}$ of DNA and is unique, except for the presence of a repetitive insertion element in the Canton-S strain.

Restriction site polymorphisms as genetic markers. Chromosomal walking provides a useful method for isolating genome regions of interest. However, one major drawback is that there exists no general method of identifying the critical sequences on the hundreds of kilobase pairs of DNA one can isolate by this method. We have solved this problem in the case of $d n c$ by mapping a $d n c$ mutation by recombination on the cloned DNA, using restriction site polymorphisms identified and localized by prior survey as genetic markers.

This analysis is limited by the number of recombinants one can select and on the frequency of restriction site polymorphism. To determine whether polymorphism is frequent enough to make this approach feasible, we surveyed the restriction sites of several different $\mathrm{X}$ chromosomes across a ca. 40-kb stretch of chromomere 3D4. The X chromosomes utilized in this study were Canton-S, $y c v v f, y w f$, and Amherst, selected because they have served as parental chromosomes for the induction of $d n c$ mutations or flanking markers (7). DNA was isolated from flies carrying these chromosomes, digested with 24 different restriction enzymes, most of which recognize hexanucleotide sequences, blotted after electrophoresis, and probed separately with $\lambda \mathrm{cDm} 2301, \lambda \mathrm{cDm} 2405$, and $\lambda \mathrm{dDm} 2500$.

Most of the altered restriction patterns observed between different $X$ chromosomes were of two types. Many were a change of one band to two smaller bands whose total length approximated that of the first. We interpret these as losses or gains of single restriction sites. A second major class showed a single new band with an altered mobility relative to the standard, suggesting a length change of plus or minus 300

\begin{tabular}{|c|c|c|c|}
\hline & ycuvf & ywf & Amherst \\
\hline CANTON-S & $3 \%$ & $7 \%$ & $4 \%$ \\
\hline ycvvf & & $8 \%$ & $6 \%$ \\
\hline ywf & & & $9 \%$ \\
\hline
\end{tabular}

FIG. 4. Percent restriction site polymorphism. The percentage of restriction sites polymorphic in chromomere 3D4 between pairs of four different chromosomes is shown. Canton-S and Amherst are two standard wild-type strains. The chromosome y $c v v f$ carries as visible markers yellow body color $(y)$, crossveinless wings $(c v)$, vermillion eyes $(v)$, and forked bristles $(f)$. The chromosome $y w f$ carries $y, f$, and white eyes $(w)$. All of these chromosomes are $d n c^{+}$.

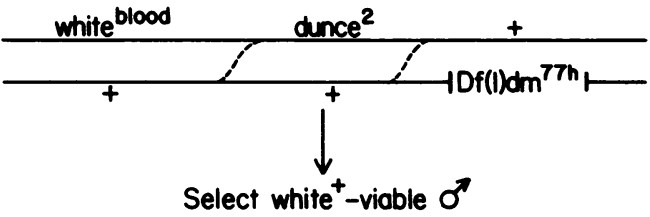

FIG. 5. Cross to map dunce $e^{2}$. Females carrying white $e^{\text {blood }}\left(w^{b l}\right)$ and dunce ${ }^{2}\left(d n c^{2}\right)$ on one homolog and heterozygous with $D f(l) d m^{77 h}$ were constructed and mated. Male progeny were scored and white ${ }^{+}$-viable males mated to attached-X females.

base pairs. These could be small insertions or deletions, or they could be losses or gains of single restriction sites near one end of the standard fragment. We would not have detected on our blots fragments of $<500$ base pairs that would indicate the latter possibility. However, for the purpose of quantitating polymorphism, this class of alteration was counted as one change. In this manner, we tabulated the number of changes between the four chromosomes indicated.

Approximately 140 restriction sites were surveyed in the Canton-S chromosome. The $y c v v f$ chromosome was closest to Canton-S, exhibiting about $3 \%$ divergence, and $y$ $w f$ was the most divergent, with about $7 \%$ of the sites different (Fig. 4). The Amherst/ $y w f$ comparison yielded a $9 \%$ polymorphic value. However, although most polymorphisms were scattered throughout the $40-\mathrm{kb}$ interval, many of the $y w f$ polymorphisms mapped to the area represented by the right side of $\lambda \mathrm{dDm} 2500$. This suggests that the $y w f$ chromosome may have a major sequence alteration in this vicinity, possibly the residence of a transposable element not found in the Canton-S chromosome. Consequently, we view the comparisons with $y w f$ as overestimates of the amount of divergence due to random base change. A 3 to $6 \%$ estimate
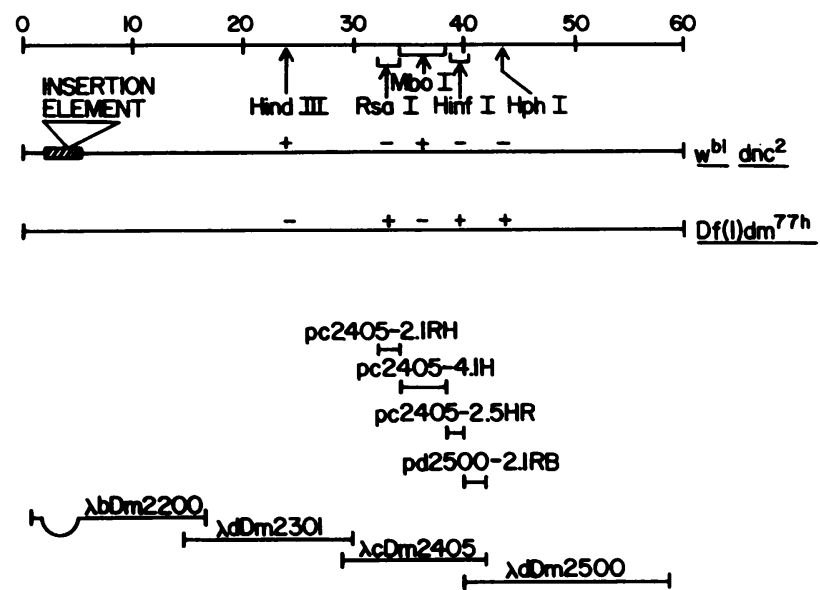

FIG. 6. Restriction site polymorphisms, locations, and probes. The coordinate system used to define the location of certain polymorphisms sets zero as the right site of the 2.2-kb EcoRI fragment of $\lambda \mathrm{dDm} 2106$ and includes unique-sequence DNA extending to the right. This coordinate system is reproduced in Fig. 2 for comparison. The polymorphisms used in this study (these are not the only ones we have detected) between the $w^{b l} d n c^{2}$ chromosome and $D f(I) \mathrm{dm}^{77 h}$ include the insertion element between coordinates 2 and 5 detected by probing genome blots with $\lambda \mathrm{bDm} 2200$, a HindIII polymorphism detected by $\lambda \mathrm{dDm} 2301$, an $R$ saI difference within the 2.1-kb subclone pc2405-2.1RH, an MboI difference within pc2405$4.1 \mathrm{H}$, a Hinfl change in pc2405-2.5HR, and an $H p h \mathrm{I}$ polymorphism detected by pd2500-2.1RB. 
of divergence between any two chromosomes is more conservative and realistic. This value is high enough to make efficient use of restriction site polymorphisms as genetic markers (see below).

Recombinational mapping of $d n c^{2}$. The female fly genotype that has produced the most informative recombinant progeny is shown in Fig. 5. One homolog carries the $d n c^{2}$ allele, a mutation which alters the kinetic properties of cyclic AMP phosphodiesterase $(7,16)$ and which is therefore a good marker for the structural portion of the gene, and the leftside visible marker white $e^{\text {blood }}\left(w^{b l}\right)$. The other homolog is the $D f(1) d m^{77 h}$ chromosome.

From the heterozygous females depicted, 126 of 7,350 male progeny scored were white ${ }^{+}\left(w^{+}\right)$. This gives a map distance between white and $D f(1) d m^{77 h}$ of 1.7 units, lower than the standard distance (20) of 3.1 between white and the diminutive $(\mathrm{dm})$ locus, located in chromomere 3D5 or 3D6 (G. LeFevre, personal communication). This depressed value possibly reflects crossover suppression due to the deficiency. Most of the $w^{+}$males were bred to establish a stock of each recombinant chromosome. DNA was then prepared from 10 male flies of each stock to analyze the spectrum of restriction sites carried by each recombinant chromosome.

Some of the polymorphisms detected in the survey described above have been employed as markers in recombination experiments. Others were sought, as the analysis pinpointed critical regions, by using additional enzymes which recognize tetranucleotide sequences and by using subclones of the $d n c^{+}$region as probes. Those utilized here were detected and mapped by genome blotting experiments with DNA from Canton-S and Amherst flies, the parental stocks of the $d n c^{2}$ and $D f(1) d m^{77 h}$ chromosomes, respectively. The $w^{b l}$ marker was introduced into the $d n c^{2}$ chromosome by recombination and potentially introduced restriction sites from a different genetic background, but the analysis presented below indicates that the crossover which produced this chromosome occurred to the left of the $d n c$ region. Figure 6 presents the polymorphic sites employed and the probes utilized to detect these differences. An arbitrary coordinate system in $\mathrm{kb}$ has been assigned to this region, starting with the right-hand site of the 2.2-kb EcoRI fragment carried by $\lambda \mathrm{dDm} 2106$, which is the approximate location of the $D f(1) N^{64 j 15}$ right breakpoint (Fig. 2).

DNA from 115 white ${ }^{+}$-viable recombinants was first analyzed for the presence or absence of the insertion element carried by the $w^{b l} d n c^{2}$ chromosome at coordinate 2 to 5 , by probing genome digests with $\lambda \mathrm{bDm} 2200$. Twenty recombinants showed the $D f(I) d m^{77 h}$ restriction pattern (Fig. 7), indicating the absence of the insertion element. Thus, the crossovers which produced these recombinants occurred to the right of the insertion element. Phenotypic analysis of these indicates that both $d n c^{+}$and $d n c^{2}$ recombinants were recovered (Table 1 ). This reveals that the $d n c^{2}$ lesion resides to the right of the insertion element.

These recombinants were then analyzed for the polymorphisms displayed in Fig. 6 by probing restriction digests with the appropriate cloned probes. We analyzed the 20 recombinants, first with respect to the HindIII polymorphism, then with respect to the $H p h I$ configuration detected by pd25002.1RB followed by analysis of the $\mathrm{MboI}$ sites in pc24054.1H. Those recombinants displaying a crossover position in the HindIII-MboI interval were then analyzed for RsaI sites; those in the MboI-HphI region were examined for HinfI restriction sites. Fig. 7 displays some of the data, and the data are summarized in Fig. 8 and Table 1. We have detected recombination in six different regions: to the left of the insertion element, between the insertion element and the $H$ indIII polymorphism, between the HindIII and RsaI polymorphisms, between the RsaI and $M b o I$ polymorphisms, between the HinfI and $H p h I$ polymorphisms, and between the $H p h I$ polymorphism and the left breakpoint of $D f(1) d m^{77 h}$. Most importantly, we have recovered one

TABLE 1. Spectrum of polymorphisms in recombinant chromosomes

\begin{tabular}{|c|c|c|c|c|c|c|c|}
\hline \multirow{2}{*}{ Recombinant $^{b}$} & \multirow{2}{*}{ Dunce phenotype $^{c}$} & \multicolumn{6}{|c|}{ Parentage of restriction sites ${ }^{a}$} \\
\hline & & HindIII at 24 & RsaI at $32-34$ & MboI at 34-38 & Hinfl at $38-41$ & HphI at 44 & Crossover region $^{d}$ \\
\hline 6 & $\mathrm{~m}$ & $D f$ & $d n c^{2}$ & $d n c^{2}$ & & $d n c^{2}$ & 3 \\
\hline 8 & + & $?$ & & $D f$ & & $D f$ & 6 \\
\hline 9 & $\mathbf{m}$ & $D f$ & $d n c^{2}$ & $d n c^{2}$ & & $d n c^{2}$ & 3 \\
\hline 10 & $\mathrm{~m}$ & $d n c^{2}$ & & $d n c^{2}$ & & $d n c^{2}$ & 2 \\
\hline 11 & $\mathrm{~m}$ & $D f$ & $D f$ & $d n c^{2}$ & & $d n c^{2}$ & 4 \\
\hline 12 & + & $D f$ & & $D f$ & $D f$ & $d n c^{2}$ & 5 \\
\hline 18 & + & $D f$ & & $D f$ & & $D f$ & 6 \\
\hline 20 & + & $D f$ & & $D f$ & & $D f$ & 6 \\
\hline 29 & + & $D f$ & & $D f$ & & $D f$ & 6 \\
\hline 33 & + & $D f$ & & $D f$ & & $D f$ & 6 \\
\hline 37 & + & $D f$ & & $D f$ & & $D f$ & 6 \\
\hline 40 & $\mathrm{~m}$ & $d n c^{2}$ & & $d n c^{2}$ & & $d n c^{2}$ & 2 \\
\hline 46 & $\mathrm{~m}$ & $d n c^{2}$ & & $d n c^{2}$ & & $d n c^{2}$ & 2 \\
\hline 48 & + & $D f$ & & $D f$ & & $D f$ & 6 \\
\hline 52 & + & $D f$ & & $D f$ & & $D f$ & 6 \\
\hline 53 & + & $D f$ & & $D f$ & & $D f$ & 6 \\
\hline 76 & + & $D f$ & & $D f$ & $D f$ & $d n c^{2}$ & 5 \\
\hline 128 & $\mathrm{~m}$ & $?$ & $d n c^{2}$ & $d n c^{2}$ & & $d n c^{2}$ & 2 or 3 \\
\hline 131 & + & $D f$ & & $D f$ & & $D f$ & 6 \\
\hline 133 & + & $D f$ & & $D f$ & & $D f$ & 6 \\
\hline
\end{tabular}

${ }^{a} d n c^{2}$, Parentage from the $w^{b l} d n c^{2}$ chromosome; $D f$, parentage from $D f(1) d m^{77 h}$

${ }^{b}$ Twenty recombinants from $w^{b l} d n c^{2} / D f(1) d m^{77 h}$ females are identified by their assigned numbers. These have arisen from crossover to the right of the insertion element but to the left of the diminutive $(\mathrm{dm})$ deficiency $77 \mathrm{~h}$ (Fig. 5).

${ }^{c}+, d n c^{+} ; \mathrm{m}, d n c^{2}$.

${ }^{d}$ Numbers correspond to regions in Fig. 8. 
A

$\begin{array}{llllllllllll}1 & 5 & 6 & 7 & 10 & 11 & 12 & 13 & 14 & 16 & 26 & 27\end{array}$

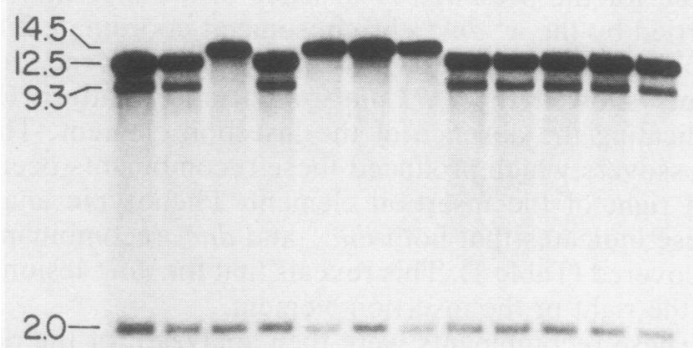

B
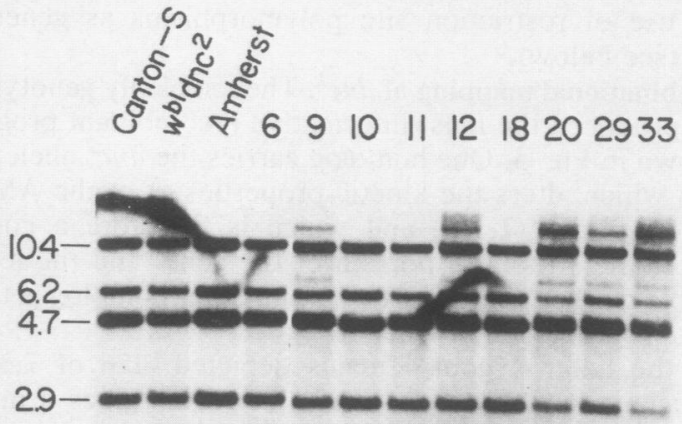

$2.1-\quad-\infty-\infty-\infty$

$1.4---$

$0.7-$

C

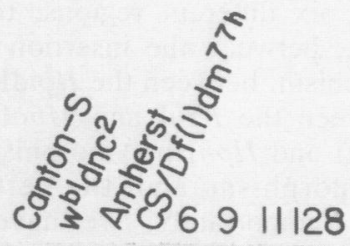

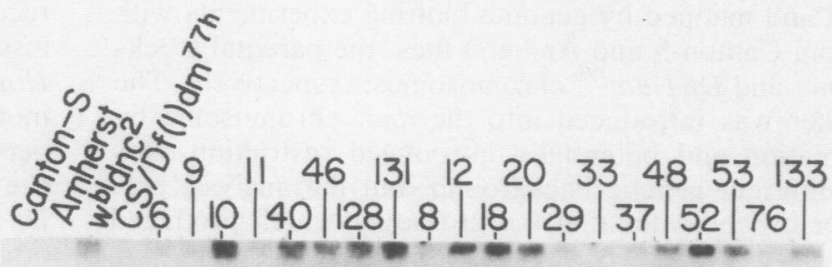

ar

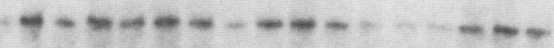

1.8
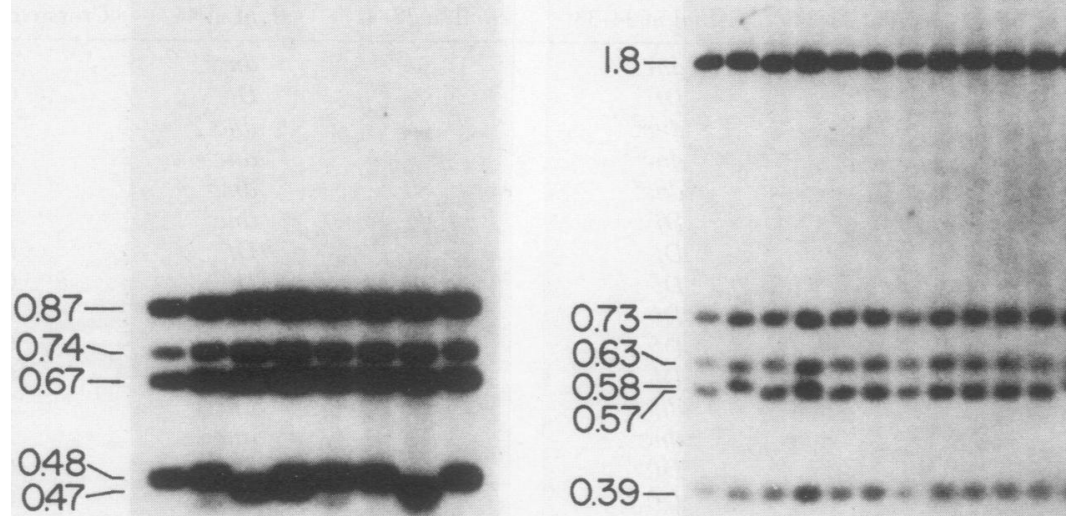

0.73- - - $0.63 \gamma$
0.58
0.57

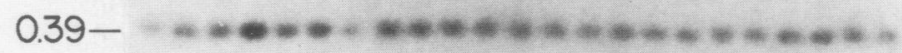

FIG. 7. A through D. 


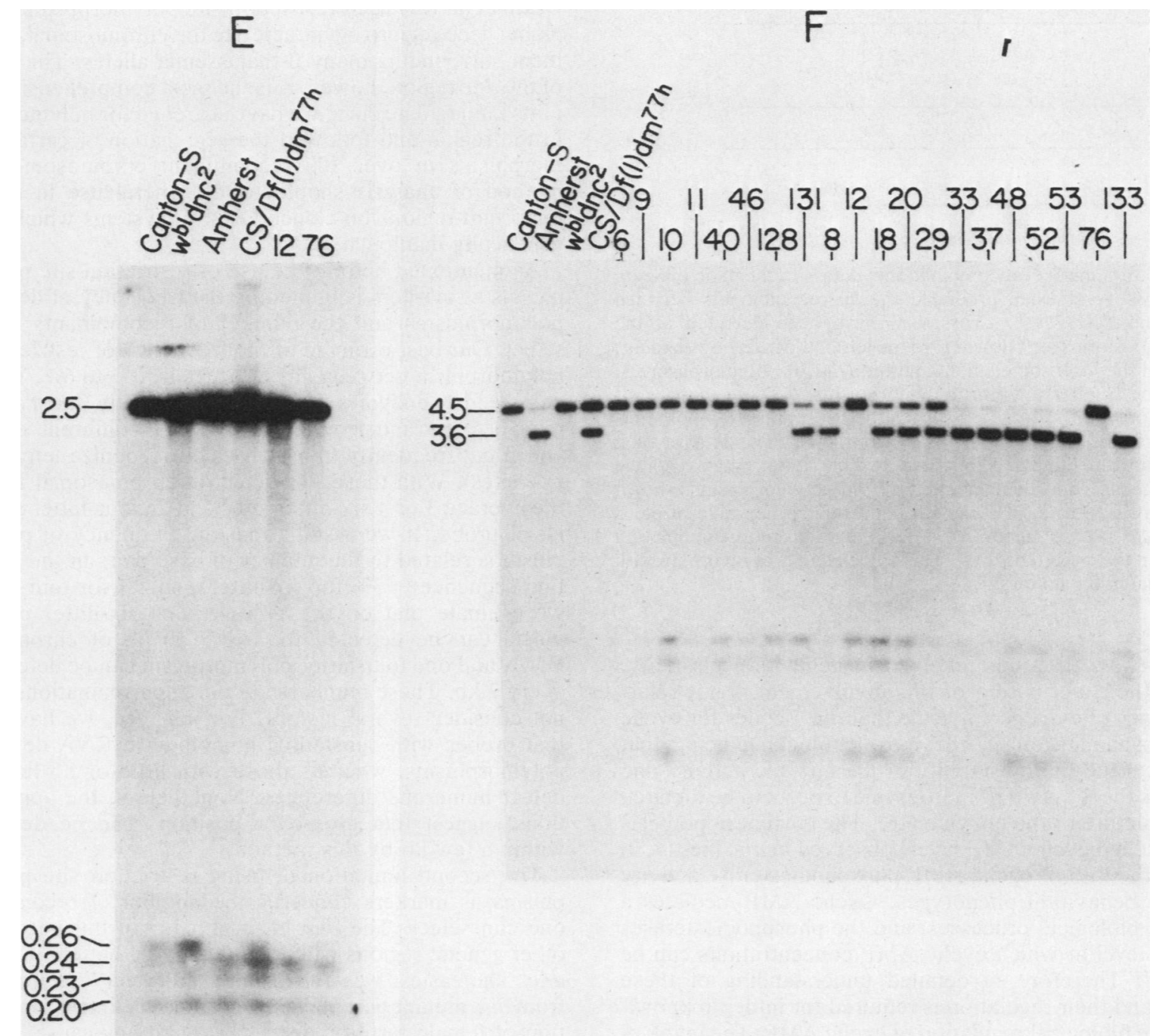

FIG. 7. Genome blots of recombinants. DNA prepared by a microprocedure (see text) was digested with restriction endonucleases, electrophoresed, blotted, and probed to detect the array of restriction sites in parental and recombinant chromosomes from the cross depicted in Fig. 5. (A) EcoRI digests probed with $\lambda b D m 2200$ to show the presence or absence of the insertion element (Fig. 6). This is 1 representative of 10 blots to probe the 115 white ${ }^{+}$-viable recombinants. Canton-S and its derivative, $w^{b l} d n c^{2}$ (not shown), show a pattern identical to that of those recombinants with fragments of $12.5,9.3$, and $2.0 \mathrm{~kb}$; Amherst and the derived chromosome $D f(1) d m^{77 h}$ show the $14.5-$ and $2.0-\mathrm{kb}$ bands (not shown). Recombinants 6, 10,11, and 12 lack the insertion element found in $w^{b l} d n c^{2}$ and therefore must have arisen as a result of crossover to the right of the element. (B) HindIII digests of several recombinants and controls after probing with $\lambda \mathrm{dDm} 2301$. The 1.4- and 0.7-kb HindIII fragments are diagnostic of Canton-S origin; the 2.1-kb fragment is diagnostic of the Amherst origin. Recombinant 10 exhibits the Canton-S pattern, and therefore, it exhibits crossover to the left of the HindIII polymorphism located at coordinate 24 . (C) $R s a$ I digests probes with pc2405-2.1RH. Canton-S (CS) exhibits a 0.48-kb Rsal fragment, and Amherst exhibits a $0.47-\mathrm{kb}$ fragment. Note that the heterozygous control, Canton-S/Df(l)dm $77 h$, shows both. (D) $M b o$ I digests of recombinants and controls probed with pc2405-4.1H. The polymorphism here is between a 0.58-kb versus a 0.57-kb MboI band. (E) Hinfl digests of recombinants and controls probed with pc2405-2.5HR, showing the presence of an 0.26-kb band in Canton-S and its absence in Amherst. (F) HphI digests. The probe is pd2500-2.1RB. The polymorphism has been mapped to a site comprising the right end of the 3.6-kb $H$ phI fragment observed in Amherst, which maps about $1 \mathrm{~kb}$ to the right of the right end of pd2500-2.1RB but is detected by this probe. The presence of the 3.6-kb band is diagnostic of the Amherst parentage. Partial digestion of an Amherst-derived chromosome results in the presence of a 4.5-kb $\mathrm{HphI}$ band in addition to the 3.6-kb band.

recombinant (number 11 ) between the $R s a I$ and $M b o$ I polymorphisms which is phenotypically $d n c^{2}$, thus showing that the $d n c^{2}$ lesion is to the right of the $R s a$ I polymorphism. In addition, two $d n c^{+}$recombinants (numbers 12 and 76) exhibited crossovers between the HinfI and $H p h I$ polymorphisms. Therefore, the $d n c^{2}$ lesion must reside to the left of the $H p h I$ polymorphism. This maps $d n c^{2}$ to the $10-$ to $12-\mathrm{kb}$ region defined physically on the left by the $R s a$ I polymorphism at coordinate 32 to 34 and the $H p h I$ polymorphism at coordinate 44.
In summary, we have generated recombinant chromosomes produced by crossover near to, but on the left and right of, the $d n c^{2}$ mutation, and we have used restriction site polymorphisms between the two parental chromosomes as genetic markers to map this lesion. The data map $d n c^{2}$ to a 10- to $12-\mathrm{kb}$ interval.

\section{DISCUSSION}

The $d n c$ gene is intriguing in several respects. The variety of behavioral changes that occur when the gene undergoes 


(1)

0.12 map units

$\sim 3 \mathrm{~kb} / 0.01$ map unit

FIG. 8. Summary of recombinational data. A schematic diagram of the crossovers which produced the 20 recombinants between $w^{b l} d n c^{2}$ and $D f(1) d m^{77 h}$. Crossovers have been detected at the numbered pcsitions as follows: 1 , to the left of the insertion element; 2, between the insertion element and the HindIII polymorphism; 3 , between the HindIII and RsaI polymorphisms; 4, between the RsaI and $M b o I$ polymorphisms; 5, between the Hinfl and HphI polymorphisms; 6 , to the right of the $H p h I$ polymorphism. The data for each recombinant are tabulated in Table 1 . The genetic map distance between the insertion element and the $H p h \mathrm{I}$ polymorphism is shown and is converted to a DNA length equivalent. The value of ca. 3 $\mathrm{kb} / 0.01$ map unit is uncorrected for the reduced recombination observed in the cross (see text). This value compares favorably with that calculated for notch (2).

mutation show that it is involved in behavioral plasticity, although the exact nature of this involvement is not clear. Several lines of evidence indicate that $d n c^{+}$codes for cyclic AMP phosphodiesterase, the most compelling being that $d n c^{l}$ affects the thermostability of the enzyme activity and $d n c^{2}$ alters the $K_{m}$, two properties one expects to be dictated by the structure of the enzyme (16). The current hypothesis is that the high cyclic AMP levels observed in $d n c$ flies $(4,7)$ owing to the loss of cyclic AMP phosphodiesterase activity cause the behavioral phenotypes. Cyclic AMP mediates a variety of biological processes, and the phosphodiesterases form one level at which cyclic AMP concentrations can be controlled. Therefore, a detailed understanding of these enzymes and their regulation is required for in-depth knowledge of the biological regulation of cyclic AMP. The involvement of the gene in behavioral processes and in cyclic AMP metabolism have provided the impetus to initiate a study of the structure, regulation, function, and evolution of the gene.

Chromosomal walking was judged to be the method of choice for isolating the gene, and it has allowed the recovery of a large region of the $\mathrm{X}$ chromosome including $d n c^{+}$. Since genetic analysis of certain chromosomal aberrations had defined the gene to chromomere 3D4, we hoped early in this study that these aberrations would limit the gene to a manageable length of DNA sequence. The analyses of the aberrations delimit $d n c^{+}$to about $50 \mathrm{~kb}$, a region twice as large as anticipated from consideration of the average DNA content per chromomere. This required us to devise an additional method for localizing $d n c$ sequences on the cloned DNA.

The problem was solved by mapping a $d n c$ mutation by recombination, with restriction site polymorphisms as genetic markers. Others have effectively used restriction fragment polymorphisms as physical markers. Steinmetz et al. (29) analyzed two recombinants arising from crossover in the $\mathrm{H}$ 2D-Tla interval to map a polymorphic fragment homologous to a transplantation antigen pseudogene to the $\mathrm{Qa}-2,3$ gene cluster. Polymorphic restriction fragments carrying $\mathrm{H}-2$ related sequences have been mapped relative to other included markers in $t$ chromosomes by recombination (27).
Orkin et al. (25) used restriction site polymorphism in the $\beta$ globin gene region to characterize the chromosomal environment surrounding many $\beta$-thalassemia alleles. The analysis of the $d n c$ region, however, is the most comprehensive study of its kind to date since we have searched for polymorphisms in the region and followed the segregation of certain polymorphisms in over 100 recombinant chromosomes. This method of analysis should be of general use in mapping important regions on cloned DNA in systems which can be genetically manipulated.

As mentioned before, the use of restriction site polymorphisms as markers is limited by the frequency of detectable polymorphisms and the number of recombinants one can select. Our best estimate of the frequency of restriction site polymorphism between any two strains is 3 to $6 \%$. We have employed 24 enzymes which cut infrequently (most of which recognize hexanucleotide sites) and 14 different enzymes which cut frequently (most of which rêcognize tetranucleotide sites). With these, we detect in chromosomal DNA on the average 3 or 4 six-hitter sites and 25 four-hitter sites per $\mathrm{kb}$ of probe. If we assume that the frequency of polymorphism is related to the number of base pairs in the recognition sequence $(\sim 3 \%$ for six-hitters, so $2 \%$ for four-hitters), we estimate that on the average, one six-hitter polymorphism can be detected for every $10 \mathrm{~kb}$ of chromosomal DNA, and one four-hitter polymorphism can be detected for every $2 \mathrm{~kb}$. These numbers are only approximations and do not consider several factors. For instance, we have noted that probes with substantial homology to RNA detect few polymorphisms, whereas those with little or no homology detect numerous differences. Nonetheless, the approximations suggest that crossover positions can be defined to within a few kb by this method.

The second limitation of using restriction site polymorphisms as markers concerns the number of recombinants one can select. The dnc locus is poor in this regard, and other genetic regions will undoubtedly be better suited than $d n c$. The easiest way for distinguishing $d n c^{+}$recombinants from $d n c$ mutant parental chromosomes is to test for restoration of female fertility since $d n c$ mutations cause sterility. We have done this for the $126 w^{+}$-viable recombinants. However, this phene is particularly sensitive to suppression effects incurred upon altering the genetic background (26; Davis and Kauvar, in press). Of the $126 w^{+}$-viable recombinants we selected, 4 stocks were fertile yet showed crossover to the left of the insertion element and were clearly $d n c^{2}$ by the cyclic AMP phosphodiesterase assay. We conclude that these have an altered genetic background which suppresses the sterility phenotype. For the 20 recombinants to the right of the insertion element, phenotypic analysis has included both fertility tests and cyclic AMP phosphodiesterase assays.

Intragenic crossovers are potentially more useful than crossovers to the left or right of an allele for gene mapping. We have obtained a surprising result by analyzing polymorphisms in putative $d n c^{+}$recombinants generated from females heterozygous for the alleles $d n c^{M 14}$ and $d n c^{2}$ (R. Davis, and H. Salz, unpublished data). The analyses of restriction site polymorphisms in the recombinants indicate that all of the sequence information within the $50-\mathrm{kb} d n c$ gene region is derived from the $d n c^{M 14}$ chromosome. That is to say, we have failed to detect any evidence of recombination at the DNA level. However, the recombinants show a major sequence alteration of the $d n c$ region, which is likely the result of an insertion element. The significance of this is not known and is under investigation. 
Finally, we have examined the coding potential of the $\mathrm{dnc}^{+}$chromosomal region, and this is the subject of a forthcoming report (manuscript in preparation). We find that the region bounded by the $R s a$ I polymorphism on the left and the $H p h I$ polymorphism on the right constitutes a single gene and that this gene produces multiple RNAs which are regulated in a complex fashion during development.

\section{ACKNOWLEDGMENTS}

We are grateful to S. Artavanis-Tsakonas, M. Muskavitch, and D. Hogness for the gift of phage clones. L. Kauvar performed cyclic AMP phosphodiesterase assays, and we are indebted to him. We thank our many colleagues for advice.

This research was supported by a grant from the National Institutes of Health (NIH) to N.D. Work conducted at Michigan State was supporte by NIH Biomedical Rasearch Support Funds and an NIH grant to R.L.D. At Caltech, R.L.D. was supported by postdoctoral fellowships from the Dampn Runyon-Walter Winchell Cancer Fund and the NIH.

\section{LITERATURE CITED}

1. Aceves-Pina, E. O., and W. G. Quinn. 1979. Learning in normal and mutant Drosophila larvae. Science 206:93-96.

2. Artavanis-Tsakonas, S., M. Müskavitch, and B. Yedvobnick. 1983. Molecular cloning of Notch, a locus affecting neurogenesis in Drosophila melanagaster. Proc. Natl. Acad. Sci. U.S.A. 80:1977-1981.

3. Booker, R., and W. G. Quinn. 1981. Conditioning of leg position in normal and mutant Drosophila. Proc. Natl. Acad. Sci. U.S.A. 78:3940-3944.

4. Byers, D., R. L. Davis, and J. A. Kiger. 1981. Defect in cyclic AMP phosphodiesterase due to the dunce mutation of learning in Drosophila melanogaster. Nature (London) 289:79-81.

5. Davidson, N., E. A. Fyrberg, N. D. Hershey, K. Kindel, R. R. Robinson, A. Sodja, and P. Yen. 1980. Recombinant DNA studies of DNA sequence organization around actin and tRNA genes of Drosophila melanogaster, p. 279-295. In S. Osawa, H. Ozeki, H. Uchida, and T. Tera (ed.), Genetics and evolution of RNA polymerase, tRNA, and ribosomes. Elsevier/North-Holland Biomedical Press, New York.

6. Davis, R. L., and J. A. Kiger. 1980. A partial characterization of the cyclic nucleotide phosphodiesterases of Drosophila melanogaster. Arch. Biochem. Biophys. 203:412-421.

7. Davis, R. L., and J. A. Kiger. 1981. dunce mutants of Drosophila melanogaster: mutants defective in the cyclic AMP phosphodiesterase enzyme system. J. Cell Biol. 90:101-107.

8. Dudai, Y. 1979. Behavioral plasticity in Drosophila mutant dunce ${ }^{D B 276}$. J. Comp. Physiol. 130:272-275.

9. Dudai, Y., Y. Jan, D. Byers, W. G. Quinn, and S. Benzer. 1976. dunce, a mutant of Drosophila deficient in learning. Proc. Natl. Acad. Sci. U.S.A. 73:1684-1688.

10. Duerr, J. S., and W. G. Quinn. 1982. Three Drosophila mutations that block associative learning also affect habituation and sensitization. Proc. Natl. Acad. Sci. U.S.A. 74:5463-5467.

11. Folkers, E. 1982. Visual learning and memory of Drosophila melanogaster wild type C-S and the mutants dunce ${ }^{\prime}$, amnesiac, turnip and rutabaga. J. Insect Physiol. 28:535-539.

12. Fyrberg, E. A., K. L. Kindle, N. Davidson, and A. Sodja. 1980. The actin genes of Drosophila: a dispersed multigene family. Cell 19:365-378.

13. Gailey, D. A., R. Jackson, and R. W. Siegel. 1982. Male courtship in Drosophila: the conditioned response to immature males and its genetic control. Genetics 102:771-782.

14. Ish-Horowicz, D., and J. F. Burke. 1981. Rapid and efficient cosmid cloning. Nucleic Acids Res. 9:2989-2998.

15. Kandel, E. R., and J. H. Schwartz. 1982. Molecular biology of learning: modulation of transmitter release. Science 218:433443.

16. Kauvar, L. M. 1982. Defective cAMP phosphodiesterase in the Drosophila memory mutant dunce. J. Neurosci. 2:1347-1358.

17. Kiger, J. A. 1977. The consequences of nullosomy for a chromosomal region affecting cyclic AMP phosphodiesterase activity in Drosophila. Genetics 85:623-628.

18. Kiger, J. A., and E. Golanty. 1977. A cytogenetic analysis of cyclic nucleotide phosphodiesterase activities in Drosophila. Genetics 85:609-622.

19. Korge, G. 1977. Direct correlation between a chromosome puff and the synthesis of a larval protein in Drosophila melanogaster. Chromosoma 62;155-174.

20. Lindsley, D. L., and E. H. Grell. 1968. Genetic variations of Drosophila melanogaster. Carnegie Institute of Washington, Washington, D.C.

21. Maniatis, T., R. C. Hardison, E. Lacy, J. Lauer, C. O'Connell, D. Quon, G. K. Sim, and A. Efstratiadis. 1978. The isolation of structural genes from libraries of eucaryotic DNA. Cell 15:687701.

22. McGinnis, W., J. Farrell, and S. Beckendorf. 1980. Molecular limits on the size of a genetic locus in Drosophila melanogaster. Proc. Natl. Acad. Sci. U.S.A. 77:7367-7371.

23. Mullins, J. I., J. W. Casey, M. O. Nicolson, K. B. Burck, and N. Davidson. 1981. Sequence arrangement and biological activity of cloned feline leukemia virus proviruses from a virus-productive human cell line. J. Virol. 38:688-703.

24. Muskavitch, M., and D. S. Hogness. 1980. Molecular analysis of a gene in a developmentally regulated puff of Drosophila melanogaster. Proc. Natl. Acad. Sci. U.S.A. 77:7362-7366.

25. Orkin, S. H., H. H. Kazazian, S. E. Antonavakis, S. C. Goff, C. D. Boehm, J. P. Sexton, P. G. Waber, and P. Giardina. 1982. Linkage of $\beta$-thalassaemia mutations and $\beta$-globin gene polymorphisms with DNA polymorphisms in human $\beta$-globin gene cluster. Nature (London) 296:627-631.

26. Salz, H. K., R. L. Davis, and J. A. Kiger. 1982. Genetic analysis of chromomere 3D4: The dunce and sperm-amotile genes in Drosophila melanogaster. Genetics 100:587-596.

27. Shin, H., J. Stavnexzer, K. Artzt, and D. Bennett. 1982. Genetic structure and origin of $t$ haplotypes of mice, analyzed with $\mathrm{H}-2$ cDNA probes. Cell 29:969-976.

28. Spofford, J. 1976. Position-effect variegation in Drosophila, p. 955-1019. In M. Ashburner and E. Novitski (ed.), Genetics and biology of Drosophila, vol. 1c. Academic Press, Inc., New York.

29. Steinmetz, M., K. W. Moore, J. G. Frelinger, B. T. Sher, F.-W. Shen, E. A. Boyse, and L. Hood. 1981. A pseudogene homologous to mouse transplantation antigens: transplantation antigens are encoded by eight exons that correlate with protein domains. Cell 25:683-692.

30. Sturtevant, A. H. 1913. The linear arrangement of six sex-linked factors in Drosophila, as shown by their mode of association. J. Exp. Zool. 14:43-59.

31. Tempel, B. L., N. Bonini, D. Dawson, and W. G. Quinn. 1983. Reward learning in normal and mutant Drosophila. Proc. Natl. Acad. Sci. U.S.A. 80:1482-1486.

32. Young, M. W. 1979. Middle repetitive DNA: a fluid component of the Drosophila genome. Proc. Natl. Acad. Sci. U.S.A. 76:6274-6278. 